\title{
THE LEBESGUE INTEGRAL AS THE ALMOST SURE LIMIT OF RANDOM RIEMANN SUMS
}

\author{
JOHN C. KIEFFER AND CASLAV V. STANOJEVIĆ
}

\begin{abstract}
A method is given for generating random intermediate points for a sequence of partitions. For the corresponding random Riemann sums it is shown that they converge almost surely to the Lebesgue integral.
\end{abstract}

1. Introduction. Various Riemann-type sums have been used for an alternative approach to the Lebesgue integral. For instance, S. Saks [1] defined abstract lower Riemann-Darboux sums to obtain an integral equivalent to the Lebesgue integral.

R. Henstock [2] introduced the concept of compatible partitions and defined an integral of the Lebesgue power in the following manner. Let $P$ be a partition of $[0,1]$, i.e. $0=x_{0}<x_{1}<\cdots<x_{n}=1$ with arbitrary $z_{j} \in\left[x_{j-1}, x_{j}\right]$. The partition $P$ is compatible with the function $\delta>0$ if for each $j=1, \ldots, n$

$$
\left|x_{j}-z_{j}\right|<\delta\left(z_{j}\right), \quad\left|z_{j}-x_{j-1}\right|<\delta\left(z_{j}\right) \text {. }
$$

The Riemann-complete integral of $f$ on $[0,1]$ is defined as a number $I(f)$ such that to each $\epsilon>0$ there corresponds a function $\delta>0$ on $[0,1]$, with

$$
\left|\sum_{j=1}^{n} f\left(z_{j}\right)\left(x_{j}-x_{j-1}\right)-I(f)\right|<\epsilon,
$$

for all sums over partitions of $[0,1]$ compatible with $\delta$. Henstock [2] proved that there exists at least one such partition. (For comparison of the Riemann-complete integral and the Lebesgue integral see [3].)

The Henstock definition of the Riemann-complete integral takes care of one of the shortcomings of the classical Riemann integral. Namely, instead of requiring the existence of the limit of Riemann sums for all partitions with vanishing norms, Henstock relaxes that requirement to only those partitions that are compatible with $\delta$.

The other shortcoming of the Riemann integral concerns the choice of the set of intermediate points. The purpose of this paper is to show how by randomizing the choice of the set of intermediate points one can get the Lebesgue integral as the almost sure limit of random Riemann sums, i.e. the Riemann sums that correspond to the random choice of the set of intermediate points. Our approach provides a method of generating those random intermediate points for a sequence of partitions of $[0,1]$.

2. Random Riemann sums. Throughout the paper we shall consider a sequence $\left\{P_{n}\right\}$ of partitions of $[0,1]$ such that each $P_{n}$ consists of finitely many subintervals,

Received by the editors August 20, 1981.

1980 Mathematics Subject Classification. Primary 28A25.

Key words and phrases. Random Riemann sums, martingales, Lebesgue integral.

(c) 1982 American Mathematical Society 0002-9939/81/0000-0758/\$03.75 
each $P_{n+1}$ is finer than $P_{n}$, and

$$
\lim _{n \rightarrow \infty} \max \left\{m(I): I \in P_{n}\right\}=0,
$$

where $m$ is the Lebesgue measure on $[0,1]$.

For each $P_{n}$ let $S_{P_{n}}$ be a set of points consisting of one point $X_{P_{n}}(I)$ from each $I \in P_{n}$. In the case of the Riemann integral the limit of the Riemann sum is required to exist for any choice of $X_{P_{n}}(I)$. We shall relax that and require $S_{P_{n}}$ to be a random set in the sense of the following definition.

DEFINITION 2.1. Let $P$ be a partition of $[0,1]$, and for each $I \in P$ let $X_{P}(I)$ be a random variable uniformly distributed over $I$. The set $S_{P}=\left\{X_{P}(I)\right\}_{I \in P}$ is called a random set of intermediate points for $P$.

Now we can randomize the Riemann sum in a formal manner.

DEFINITION 2.2. Let $S_{P}$ be the random set of intermediate points for $P$. The sum

$$
\sum_{I \in P} f\left(X_{P}(I)\right) m(I)
$$

is called a random Riemann sum for the real-valued function $f$ on $[0,1]$.

It is easy to show (we leave this to the reader) that if for each $n$ we have a random set $S_{n}=\left\{X_{P_{n}}(I)\right\}$ of intermediate points for $P_{n}$, then for every Lebesgue integrable $f$ on $[0,1]$, the sequence of random Riemann sums $\left\{\sum_{I \in P_{n}} f\left(X_{P_{n}}(I)\right) m(I)\right\}$ converges in $L^{1}$-norm to $\int_{0}^{1} f d m$ as $n \rightarrow \infty$.

In the rest of the paper we shall show that there exists a sequence of random sets of intermediate points such that the random Riemann sums converge almost surely as well as in $L^{1}$. To obtain the almost sure convergence we shall make use of the well-known theorem of Doob [4] (see also [5, Theorem 7.6.3]) concerning the convergence of reverse martingales.

3. Convergence of random Riemann sums. The proof of our main result (alluded to above) has three essential parts. First we shall show that on some probability space one can construct for each $n$ a set of random variables $S_{n}=\left\{X_{P_{n}}(I): I \in\right.$ $\left.P_{n}\right\}$ such that both of the following hold:

(a) each $S_{n}$ is a random set of intermediate points for $P_{n}$;

(b) for each Lebesgue integrable $f,\left\{\sum_{I \in P_{n}} f\left(X_{P_{n}}(I)\right) m(I)\right\}$ forms a reverse martingale.

The second part is to observe that because of (a), the sums in (b) converge in $L^{1}$ norm to $\int_{0}^{1} f$. The third and last part is to observe that because of the reverse martingale property (b), the convergence of the sums in (b) to $\int_{0}^{1} f$ must be almost sure.

Here is our main result.

THEOREM. On some probability space there exists a sequence of sets $\left\{S_{n}\right\}$ such that $S_{n}$ is a random set $S_{P_{n}}$ of intermediate points for the partition $P_{n}, n=1,2, \ldots$, and for every Lebesgue integrable function $f$ on $[0,1]$, the random Riemann sums

$$
\left\{\sum_{I \in P_{n}} f\left(X_{P_{n}}(I)\right) m(I)\right\}
$$

converge almost surely to $\int_{0}^{1} f d m$. 
PROOF. First we construct $S_{1}$ as $S_{P_{1}}=\left\{X_{P_{1}}(I)\right\}_{I \in P_{1}}$, where the random variables $X_{P_{1}}(I)$ are independent and each uniformiy distributed over $I$.

Then for each $n$ we define the mapping $\phi_{n}: P_{n+1} \rightarrow P_{n}$ such that $\phi_{n}(J) \supset J$ where $J \in P_{n+1}$. Now we proceed by induction. Given

$$
S_{P_{1}}=S_{P_{1}}^{*}, \ldots, S_{P_{n}}=S_{P_{n}}^{*},
$$

choose $S_{P_{n+1}}$ such that the random variables

$$
\left\{X_{P_{n+1}}(J): X_{P_{n}}^{*}\left(\phi_{n}(J)\right) \notin J\right\}
$$

are conditionally independent, each $X_{P_{n+1}}(J)$ in (3.1) having conditional distribution equal to the uniform distribution over $J$, and that the remaining intermediate points are defined conditionally by

$$
X_{P_{n+1}}(J)=X_{P_{n}}^{*}\left(\phi_{n}(J)\right), \quad X_{P_{n}}^{*}\left(\phi_{n}(J)\right) \in J .
$$

To express this more formally consider two sets of intervals

$$
T=\left\{J \in P_{n+1}: X_{P_{n}}^{*}\left(\phi_{n}(J)\right) \notin J\right\}, \quad \tilde{T}=\left\{J \in P_{n+1}: X_{P_{n}}^{*}\left(\phi_{n}(J)\right) \in J\right\},
$$

and let $B_{J}$ be a Borel subset of $J, J \in P_{n+1}$. Then

$$
\begin{gathered}
\operatorname{Prob}\left[X_{P_{n+1}}(J) \in B_{J}, J \in P_{n+1} \mid S_{P_{1}}=S_{P_{1}}^{*}, \ldots, S_{P_{n}}=S_{P_{n}}^{*}\right] \\
=\prod_{J \in T} m_{J}\left(B_{J}\right) \prod_{J \in \tilde{T}} \chi_{B_{J}}\left(X_{P_{n}}\left(\phi_{n}(J)\right)\right)
\end{gathered}
$$

where $m_{J}$ is the normalized Lebesgue measure on $J$, and $\chi$ denotes the characteristic function.

For fixed $n$ let $I \in P_{n}$, and fix $B_{J} \subset J$ for each $J \subset I, J \in P_{n+1}$. Then we define the event $A$ as follows:

$$
\left\{X_{P_{n+1}}(J) \in B_{J}\left(J \subset I, J \in P_{n+1}\right)\right\} .
$$

For every Lebesgue integrable function $f$ on $[0,1]$, from (3.1) and (3.2) we have

$$
\begin{aligned}
& E\left[\chi_{A} f\left(X_{P_{n}}(I)\right)\right] \\
& =\sum_{J \subset I, J \in P_{n+1}} E\left[f\left(X_{P_{n}}(I)\right) \chi_{\left\{X_{P_{n}}(I) \in B_{J}\right\}}\right] \prod_{J^{\prime} \neq J} m_{J^{\prime}}\left(B_{J^{\prime}}\right),
\end{aligned}
$$

where $E[\cdot]$ denotes the expectation.

From (3.4) and our construction we can obtain inductively these two conclusions:

(3.5) $\left\{X_{P_{n}}(I)\right\}_{I \in P_{n}}$ are independent for all $n$, and

(3.6) the random variables $X_{P_{n}}(I)$ are uniformly distributed over $I$, for all $n$ and all $I \in P_{n}$.

We notice that from (3.3) and (3.5) it follows that $I \in P_{n}$ implies that $X_{P_{n}}(I)$, $\left\{X_{P_{n+1}}(J): J \subset I\right\}$ and $S_{P_{n+1}}$ form a Markov chain.

Let $f$ be a fixed Lebesgue integrable function on $[0,1]$. Then using (3.6) we get

(3.7) the sequence of random Riemann sums $\left\{\sum_{I \in P_{n}} f\left(X_{P_{n}}(I)\right) m(I)\right\}$ converges in $L^{1}$-norm to $\int_{0}^{1} f d m$.

Our next objective is to show that

$$
E\left[f\left(X_{P_{n}}(I)\right) \mid\left\{X_{P_{n+1}}(J): J \subset I\right\}\right]=\sum_{J \subset I} \frac{m(J)}{m(I)} f\left(X_{P_{n+1}}(J)\right) .
$$


Consider again the event $A$ defined in (A). If $I \in P_{n}$, it follows from (3.5) and (3.6) that

$$
\begin{aligned}
E\left[\chi_{A} \sum_{J \subset I} \frac{m(J)}{m(I)}\right. & \left.f\left(X_{P_{n+1}}(J)\right)\right] \\
= & \sum_{J \subset I}\left\{\frac{m(J)}{m(I)} E\left[f\left(X_{P_{n+1}}(J)\right) \chi_{\left\{X_{P_{n+1}}(J) \in B_{J}\right\}}\right]\right\} \prod_{J^{\prime} \neq J} m_{J^{\prime}}\left(B_{J^{\prime}}\right) .
\end{aligned}
$$

Since

$$
\frac{m(J)}{m(I)} E\left[f\left(X_{P_{n+1}}(J)\right) \chi_{\left\{X_{P_{n+1}}(J) \in B_{J}\right\}}\right]
$$

is equal to

$$
E\left[f\left(X_{P_{n}}(I)\right) \chi_{\left\{X_{P_{n}}(I) \in B_{J}\right\}}\right]
$$

statement (3.8) follows from an application of (3.4).

From the fact that $\left\{S_{P_{n}}\right\}$ form a Markov chain and from (3.8) we obtain the reverse martingale property

$$
E\left[\sum_{I \in P_{n}} f\left(X_{P_{n}}(I)\right) m(I) \mid S_{P_{n+1}}, S_{P_{n+2}}, \ldots\right]=\sum_{I \in P_{n+1}} f\left(X_{P_{n+1}}(I)\right) m(I) .
$$

Using (3.7) and recalling Doob's result [4] that a reverse martingale converges almost surely we complete the proof of the theorem.

In a similar fashion we can generate random Riemann-Stieltjes sums which converge almost surely to a given Lebesgue-Stieltjes integral. As a matter of fact we can approximate an expectation on any probability space by appropriate random sums.

\section{REFERENCES}

1. S. Saks, Theory of the integral, Monografije Matematyczne, Tom VII, Warszawa-Lwow, 1937.

2. R. Henstock, A Riemann-type integral of Lebesgue power, Canad. J. Math. 20 (1968), 78-87.

3. E. S. Smith, A comparison of the Riemann-complete and Lebesgue integrals, Eleutheria 2 (1979), 493-499.

4. J. L. Doob, Regularity properties of certain families of chance variables, Trans. Amer. Math. Soc. 47 (1940), 455-486.

5. R. B. Ash, Real analysis and probabilities, Academic Press, New York, 1972.

Department OF MAthematics, University of Missouri-Rolla, Rolla, MISSOURI 65401 